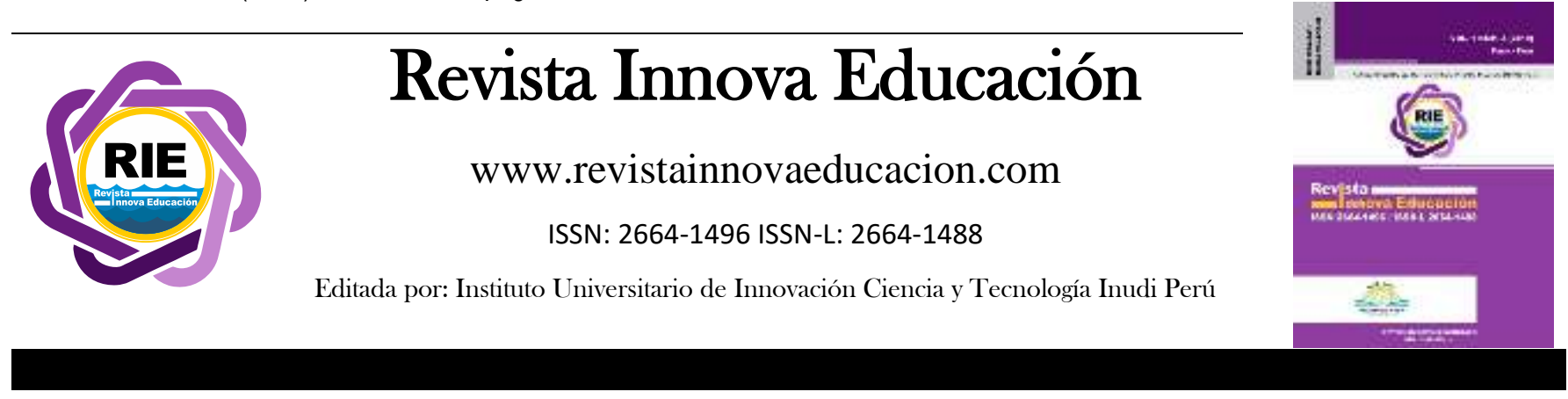

\title{
Síndrome de Burnout y desempeño laboral en el personal de salud
}

\author{
Burnout syndrome and job performance in health personnel \\ Claudia Lauracio ${ }^{1}$ \\ Universidad Nacional del Altiplano de Puno, Puno-Puno, Perú \\ (D) https://orcid.org/0000-0003-1393-0728 \\ Teófilo Lauracio \\ Universidad José Carlos Mariátegui, Moquegua-Moquegua, Perú \\ (D) https://orcid.org/0000-0002-8095-6031
}

DOI: https://doi.org/10.35622/j.rie.2020.04.003

Recibido 05/06/2020/ Aceptado 21/09/2020 Publicado 06/10/2020

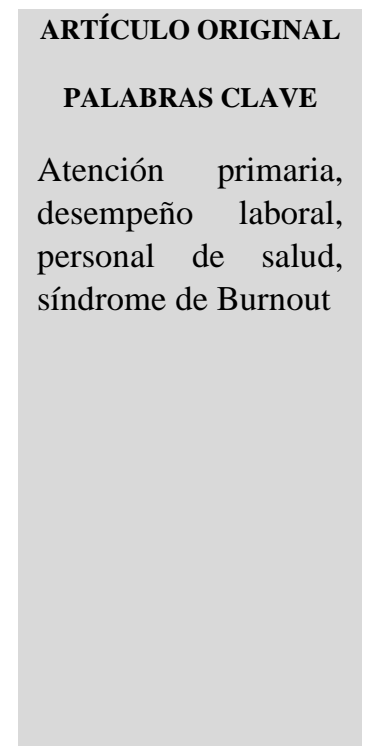

KEYWORDS

Primary care, job performance, health personnel, Burnout syndrome
Los profesionales de salud son los más vulnerables a factores que desencadenan estrés laboral asociado al síndrome de Burnout, que suele afectar a su desempeño laboral y a la calidad de su atención, ocasionando frecuentes reclamos por parte de los usuarios, en particular en el primer nivel de atención del sistema de salud. Objetivo: Identificar la presencia del síndrome de Burnout en sus 3 dimensiones: agotamiento emocional, despersonalización, realización personal, identificar el nivel de desempeño laboral en sus cuatro escalas: calidad técnica y atención médica, capacitación y educación continua, productividad, aptitudes; y establecer si hay relación entre el síndrome de Burnout y el desempeño laboral en el personal de salud del Centro de Salud Vallecito I-3, Puno (Perú). Método: Descriptivo-correlacional, la muestra estuvo constituida por el total de trabajadores del establecimiento de salud. Los instrumentos fueron el test Maslach Burnout Inventory y el cuestionario de autoevaluación del desempeño laboral. Resultados: Síndrome de Burnout 7.09 en una escala de 1 a 10 (s=2.32), desempeño laboral 35.71 en una escala de 0 a 40 ( $\mathrm{s}=0.81)$, no existe correlación entre ambas variables ( $\mathrm{r}=0.063)$. Conclusión: Se concluye que el síndrome de Burnout es de nivel medio, el desempeño laboral es alto y que no se establece relación entre el síndrome de Burnout y el desempeño laboral del personal de salud.

Health professionals are the most vulnerable to factors that trigger work stress associated with Burnout syndrome, which usually affects their work performance and the quality of their attention, causing frequent complaints from users, particularly at the first level of attention at health system centers. Objective: To identify the presence of the Burnout syndrome in its 3 dimensions: emotional exhaustion, depersonalization, personal fulfillment to identify the level of work performance in its four scales: technical quality and medical attention, training and continuing education, productivity, skills; and to establish whether there is a relation between Burnout

${ }^{1}$ Correspondencia: jclaudia67@gmail.com 
syndrome and work performance in health personnel at the Vallecito I-3 Health Center, Puno (Peru). Method: Descriptive-correlational, the sample consisted of the total number of workers in the health establishment. The instruments were the Maslach Burnout Inventory test and the job performance self-assessment questionnaire. Results: Burnout Syndrome 7.09 on a scale of 1 to $10(\mathrm{~s}=2.32)$, work performance 35.71 on a scale of 0 to $40(\mathrm{~s}=0.81)$, there is no correlation between both variables $(\mathrm{r}$ $=0.063)$. Conclusion: It is concluded that the Burnout syndrome reaches medium level, the work performance is high and there is no relationship established between the Burnout syndrome and the work performance of health personnel.

\section{INTRODUCCIÓN}

En la actualidad el estrés forma parte de nuestra vida y suele repercutir en el quehacer profesional y personal (Ortega \& López, 2016); considerado como «el malestar de nuestra civilización» (Rubio, 2016). Afecta a la salud y al bienestar personal como a la satisfacción laboral y colectiva (Terrones et al., 2016). Para la Organización Internacional del Trabajo los trabajadores de la salud son los más propensos a padecer estrés laboral, debido a las condiciones de trabajo (Gómez, 2017; Martinez, 2013; Quirate et al., 2017).

El síndrome de Burnout asociado a condiciones de estrés se desarrolla por situaciones laborales de alta implicación como ocurre con los profesionales de salud (Cavero et al., 2017; Thomaé et al., 2015). Las investigaciones confirman que este síndrome afecta más a aquellas profesiones que requieren un contacto directo con las personas y una «filosofía humanística» en el trabajo (Quirate et al., 2017; Yaya et al., 2018), pues necesitan altas dosis de entrega e implicación (Do Carmo et al., 2016). Los profesionales de la salud enfrentan a diario extensas jornadas laborales, que traen consigo enfermedades de tipo físico e incluso mental (Ayquipa, 2017).

La Red Mundial de Salud Ocupacional de la OMS, describe al síndrome de Burnout como un desgaste laboral progresivo que comienza con niveles excesivos y prolongados de estrés laboral (Graue et al., 2019), evidenciado por la irritabilidad, cansancio y tensión (Lavy \& Magdalena, 2017; Ochante et al., 2018). Por lo general, un mal día se considera un problema personal (Arias \& Muñoz, 2016); sin embargo, quizás sea síntoma de alguna disfunción o enfermedad (Sánchez \& Sierra, 2014). El síndrome de Burnout puede considerarse un problema de salud pública por sus implicancias indeseables para el empleador como para el empleado (Mansilla \& Favieres Alejandra, 2016).

La atención de calidad es relevante en los diferentes niveles de servicios de salud y está íntimamente relacionada a las altas expectativas de los usuarios (Castro et al., 2016), que en muchas ocasiones no se alcanzan; lo cual se evidencia a través de reclamos o quejas hacia el trato del personal y al servicio recibido (Ortega \& López, 2016). Existen una serie de factores laborales que causan problemas de estrés laboral, cuando se olvida el papel preponderante del recurso humano como eje del Sistema de Salud (Arias \& Muñoz, 2016; Curiel et al., 2014; Muñoz et al., 2019). Este descuido está repercutiendo en el ámbito personal, pero en gran medida en el desempeño laboral y en la calidad de atención. 
El deterioro en el desempeño laboral del profesional de salud está determinado por condiciones individuales y organizacionales se manifiesta a través de la disminución en el logro de las metas inherentes a su puesto de trabajo (Castillo et al., 2015); ausentismos, irritabilidad, baja motivación, maltrato hacia los pacientes y malas relaciones con sus compañeros; ocasionando costos adicionales a las instituciones de salud (Contreras et al., 2015; Leal et al., 2015; Vivanco et al., 2018).

Un sistema de salud óptimo se sustenta en el desempeño laboral favorable de sus trabajadores; sin embargo, los sistemas de salud han descuidado este aspecto exigiendo únicamente el cumplimiento de metas a corto plazo que conlleva una entrega laboral alta del profesional de salud (Aranda, 2014; Caballero et al., 2014; Vásquez et al., 2015), sobretodo en el primer nivel de atención donde las actividades preventivo-promocionales condicionan un contacto continuo y personalizado con la población asignada (Yslado et al., 2013; Cortez \& García, 2011; Solís et al., 2017), así como sobrecarga laboral por la amplia demanda de atención en este nivel considerado como "la puerta de entrada" al sistema de salud (Del Río et al., 2015; Díaz \& Machuca, 2018; Quiroz, 2014; Hernández, 2014).

Corresponde a las instituciones de salud proteger a sus trabajadores contra los factores que los desestabilizan física y emocionalmente, mejorando sus condiciones laborales (Ayquipa, 2017; Mejía, 2019). Al parecer desde los mismos ejes rectores del sistema de salud se está perdiendo progresivamente la humanización, impidiendo de esta manera el desarrollo de atenciones con calidad y calidez (Sostena, 2015; Consuelo, 2018 ; Vásquez et al., 2014).

El síndrome de Burnout afecta considerablemente al desempeño laboral por condicionar una baja productividad, inadecuado clima laboral, falta de cooperación y ausencia de trabajo interdisciplinario del equipo de salud (Carillo et al., 2017; Chung \& Salas, 2018; Do Carmo et al., 2016). Germen de las frecuentes quejas, desconfianza y malestar de los usuarios (Mamani et al., 2017).En ello radica la importancia de este trabajo, pues al parecer el síndrome de Burnout es considerado un problema de Salud Pública (Castillo et al., 2015; Del Río et al., 2015; Díaz \& Machuca, 2018; Hernández, 2015; Mamani et al., 2017; Thomaé et al., 2015). Este artículo pretende contribuir a su análisis, al relacionar el síndrome de Burnout con el desempeño laboral del personal de un establecimiento de salud del primer nivel de atención.

\section{MÉTODO Y MATERIALES}

Los objetivos de esta investigación fueron identificar la presencia del síndrome de Burnout en el personal de salud en sus 3 dimensiones: agotamiento emocional, despersonalización, realización personal, identificar el nivel de desempeño laboral del personal de salud en sus cuatro escalas: calidad técnica y atención médica, capacitación y educación continua, productividad, aptitudes; y establecer si hay relación entre el síndrome de Burnout y el desempeño laboral del personal de salud.

Se llevó a cabo una investigación descriptiva - correlacional. El estudio se ha realizado en la población de trabajadores del Centro de salud Vallecito I-3 - Puno, conformado por 21 personas entre médicos, odontólogos, enfermeras, obstetras, biólogos, trabajadores sociales y no profesionales como técnicos de enfermería. 
La información se recogió mediante dos cuestionarios, uno para medir el síndrome de Burnout y el otro para evaluar el desempeño laboral. El primero con el cuestionario Maslach Burnout Inventory (MBI) por su alta consistencia interna, gran aceptación internacional, evidencia de validez concurrente y divergente; y una fiabilidad cercana al 90\% diseñado por Maslach, C. y Jackson, S. en el año 1981 aplicado y validado a contextos asistenciales (Hederich-Martínez \& Caballero-Domínguez, 2016). Consta de 22 enunciados sobre los sentimientos y pensamientos relacionados a la interacción con el trabajo: 9 relacionados con recursos emocionales y cansancio, 5 con despersonalización y 8 con competencia y eficacia en el trabajo (Vivanco et al., 2018), la retroalimentación se adecua al tipo Likert. Para el segundo se empleó el cuestionario de autoevaluación del desempeño laboral un cuestionario diseñado por Valdivia, M. (Valdivia, 2014) cuyo coeficiente de Kuder Richardson: 0.728 denota alto grado de confiabilidad y consistencia interna. Este instrumento consta de 21 ítems agrupados con respuestas dicotómicas, que comprende calidad y técnica de atención, capacitación y educación continua, productividad y aptitudes.

\section{RESULTADOS}

Los resultados de la evaluación de la presencia de síndrome de Burnout en el personal de salud del C.S. Vallecito I-3, se presentan en la Tabla $\mathrm{N}^{\circ} 1$. Se aprecia que el $71.4 \%$ el grupo no profesional y el $57.2 \%$ de los profesionales presentan bajo nivel de agotamiento emocional, pareciera que no les molesta la exigencia laboral; en conjunto no hay clara inclinación en cuanto a despersonalización; pero si en realización personal el 7.6\% de los profesionales consideran que su realización personal es baja. Es algo menos entre los no profesionales: $28.6 \%$ en la escala inferior y $42.8 \%$ en la intermedia. Ello puede repercutir en la atención que brindan y la calidad de servicios que prestan, ya que los resultados óptimos se obtienen con un personal motivado y realizado personalmente (Castillo et al., 2015; Mendoza, 2016; Yslado et al., 2014). El promedio del indicador del síndrome de Burnout es 7.09 en una escala de 1 a 10 con una desviación estándar de 2.32; es decir, este personal sufre más que medianamente el síndrome de Burnout.

TABLA 1

Sindrome de Burnout en el personal de salud

\begin{tabular}{|c|c|c|c|c|c|c|}
\hline \multirow[t]{2}{*}{$\begin{array}{c}\text { SUBESCALA } \\
\text { SB } \\
\end{array}$} & \multicolumn{2}{|c|}{$\begin{array}{c}\text { AGOTAMIENTO } \\
\text { EMOCIONAL }\end{array}$} & \multicolumn{2}{|c|}{ DESPERSONALIZACIÓN } & \multicolumn{2}{|c|}{$\begin{array}{l}\text { REALIZACIÓN } \\
\text { PERSONAL }\end{array}$} \\
\hline & PROFESIONAL & $\begin{array}{c}\text { NO } \\
\text { PROFESIONAL }\end{array}$ & PROFESIONAL & $\begin{array}{c}\text { NO } \\
\text { PROFESIONAL }\end{array}$ & PROFESIONAL & $\begin{array}{c}\text { NO } \\
\text { PROFESIONAL }\end{array}$ \\
\hline BAJO & $57.2 \%$ & $71.4 \%$ & $42.9 \%$ & $28.6 \%$ & $78.6 \%$ & $28.6 \%$ \\
\hline MEDIO & $35.7 \%$ & $28.6 \%$ & $42.9 \%$ & $28.6 \%$ & $0 \%$ & $42.8 \%$ \\
\hline ALTO & $7.1 \%$ & $0 \%$ & $14.2 \%$ & $42.8 \%$ & $21.4 \%$ & $28.6 \%$ \\
\hline
\end{tabular}

Fuente: Elaboración propia

Los resultados de la evaluación del desempeño laboral del personal de salud del C.S. Vallecito I-3 se presentan en la tabla $\mathrm{N}^{\circ} 2$ 
TABLA 2

Desempeño laboral en el personal de salud

\begin{tabular}{|c|c|c|c|c|c|c|c|c|}
\hline \multirow{3}{*}{$\begin{array}{l}\text { ESCALAS } \\
\text { DL } \\
\text { SI }\end{array}$} & \multicolumn{2}{|c|}{$\begin{array}{c}\text { CALIDAD Y } \\
\text { TÉCNICA DE } \\
\text { ATENCIÓN }\end{array}$} & \multicolumn{2}{|c|}{$\begin{array}{c}\text { CAPACITACIÓN Y } \\
\text { EDUCACIÓN } \\
\text { CONTINUA }\end{array}$} & \multicolumn{2}{|c|}{ PRODUCTIVIDAD } & \multicolumn{2}{|c|}{ APTITUDES } \\
\hline & PROF. & $\begin{array}{l}\text { NO } \\
\text { PROF. }\end{array}$ & PROF. & $\begin{array}{l}\text { NO } \\
\text { PROF. }\end{array}$ & PROF. & $\begin{array}{l}\text { NO } \\
\text { PROF. }\end{array}$ & PROF. & $\begin{array}{l}\text { NO } \\
\text { PROF. }\end{array}$ \\
\hline & $70 \%$ & $68 \%$ & $41 \%$ & $25 \%$ & $84 \%$ & $68 \%$ & $77 \%$ & $57 \%$ \\
\hline NO & $30 \%$ & $31 \%$ & $59 \%$ & $75 \%$ & $16 \%$ & $32 \%$ & $23 \%$ & $43 \%$ \\
\hline
\end{tabular}

Nota: PROF. $=$ profesional; NO PROF. $=$ no profesional.

Fuente: Elaboración propia

En cuanto a la calidad y técnica de atención, el $70 \%$ de los profesionales se identifican como trabajadores que se desempeñan con eficiencia, integran diagnósticos, participan en comités de ética, realizan trabajo en equipo y certifica su profesión; en la escala de capacitación y educación continua (Salas et al., 2015), el 75\% de los no profesionales reconocen que no acuden a cursos de actualización, ni aplican guías prácticas establecidas por el eje rector; en la escala de productividad el $84 \%$ del grupo profesional, muestra que se ajusta a las necesidades de los servicios que brinda con resultados óptimos, de manera similar en la escala de aptitudes el $77 \%$ de los profesionales demuestran que realizan estrategias y adaptaciones para brindar respuestas favorables a las necesidades, muestran compromiso con su institución, brindan una atención de calidad y oportuna a los usuarios de los servicios de salud (Chumpitaz, 2017). En general el desempeño laboral del personal de salud es alto y homogéneo: 35.71 en una escala de 0 a 40, con una desviación estándar muy baja (0.81).

El análisis comparativo revela que el grupo profesional presenta una media de 7.57 del síndrome, superior al grupo no profesional que presenta una media de 6.14; ambos tienen una desviación estándar semejante. En cuanto al desempeño laboral, ambos grupos revelan desempeñarse de manera similar (ver tabla $\mathrm{N}^{\circ} 3$ ), aunque hay mayor homogeneidad en el grupo profesional con 0.67 respecto a 1.1 al grupo no profesional.

TABLA 3

Estadísticos descriptivos del síndrome de Burnout y desempeño laboral en el personal de salud

\begin{tabular}{lccccc}
\hline & $N$ & Mínimo & Máximo & Media & Desviación estándar \\
\hline Síndrome Burnout Profesionales & 14 & 3,00 & 10,00 & 7,5714 & 2,37663 \\
Desempeño laboral profesionales & 14 & 7,50 & 10,25 & 8,9000 &, 67767 \\
Síndrome Burnout no Profesionales & 7 & 4,00 & 9,00 & 6,1429 & 2,03540 \\
$\begin{array}{l}\text { Desempeño laboral no } \\
\text { profesionales }\end{array}$ & 7 & 7,75 & 11,25 & 9,0000 & 1,09924 \\
\hline
\end{tabular}

Fuente: Elaboración propia

No se ha logrado evidenciar una correlación significativa entre el síndrome de Burnout y desempeño laboral (ver tabla $\mathrm{N}^{\circ} 4$ ). El coeficiente de correlación es 0.063 , este resultado puede asociarse a la heterogeneidad de los grupos o al tipo de instrumento empleados, que deberán motivar nuevos trabajos de investigación. 
TABLA 4.

Sindrome de Burnout y desempeño laboral en el personal de salud

\begin{tabular}{|c|c|c|c|}
\hline & & $\begin{array}{l}\text { Síndrome de } \\
\text { Burnout }\end{array}$ & $\begin{array}{l}\text { Desempeño } \\
\text { Laboral }\end{array}$ \\
\hline \multirow{3}{*}{$\begin{array}{l}\text { Síndrome } \\
\text { Burnout }\end{array}$} & Correlación de Pearson & 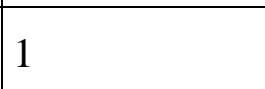 &, 063 \\
\hline & Sig. (bilateral) & &, 786 \\
\hline & $\mathrm{N}$ & 21 & 21 \\
\hline \multirow{3}{*}{$\begin{array}{l}\text { Desempeño } \\
\text { Laboral }\end{array}$} & Correlación de Pearson &, 063 & 1 \\
\hline & Sig. (bilateral) & ,786 & \\
\hline & $\mathrm{N}$ & 21 & 21 \\
\hline
\end{tabular}

Fuente: Cuestionario MBI, Cuestionario de autoevaluación del Desempeño laboral

\section{DISCUSIONES}

El síndrome de Burnout en el personal de salud es de un nivel medio, al realizar el análisis diferenciado se evidencia que es mayor en el grupo profesional respecto al no profesional, considerándose como un establecimiento de salud que presta servicios altamente profesionalizados por tener un mayor porcentaje de personal profesional. Estos resultados aportan nuevos datos en relación a estudios previos realizados en nuestro país sobre la presencia de este síndrome. Quiroz \& Saco (2016) al evaluar al personal de salud del Hospital Nacional de ESSALUD del Cusco encontraron niveles bajos de Burnout y un pequeño grupo con grado medio, resultados que se asociarían al entorno laboral, ya que un establecimiento de salud como Vallecito, tiene marcadas limitaciones en relación al personal, por lo que la carga laboral es mayor en comparación a instituciones con mayor número de trabajadores y mejor organización como un Hospital.

En la evaluación de las tres subescalas del síndrome de Burnout, resalta los hallazgos en la segunda con un nivel medio y alto que evidencia la presencia del síndrome en el personal; sin embargo para algunos autores los datos más significativos a considerar como componente central y de mayor valor para el diagnóstico del síndrome y la toma de medidas posteriores son los de la primera subescala de agotamiento emocional (Curiel et al., 2014; Hernández, 2015; Quiroz \& Saco, 2014); en contraste, la presente investigación no muestra valores importantes en esta subescala; así como en estudios similares donde valores altos en una o más subescalas indican la presencia del síndrome (Gomero, Palomino, Ruíz, \& Yesán, 2005).

Basado en este criterio tenemos el estudio realizado por Gomero, Palomino, Ruíz, \& Yesán (2005) con resultados importantes en la segunda subescala, que afirma de manera concluyente la presencia del síndrome en el personal; cabe mencionar que dicho estudio fue realizado en una institución privada, con un ambiente laboral distinto al de una institución del estado que se caracteriza por una mayor afluencia de pacientes, repercutiendo en la forma de atención y en la subescala de despersonalización (Mendoza, 2014).

Leal et al. (2015) encontró valores altos en la subescala de realización personal en profesionales de salud especialistas que difiere del presente estudio debido a que por razones 
laborales y/o familiares muchos de los profesionales de este establecimiento no pudieron continuar estudios de especialización afectando su sentimiento de realización personal (Arias \& Muñoz, 2016; Rojas et al., 2016). Miranda, Monzalvo, Hernández, \& Ocampo (2016) evaluó personal de salud en una institución privada, donde la subescala de realización personal también presentó niveles bajos, en las otras subescalas los valores fueron altos.

Mendoza H. (2012), en relación a la escala de cansancio emocional encontró niveles altos en el personal de 25 - 34 años diferente a esta investigación con niveles bajos $56.3 \%$ diferencia que se asocia a que el C.S. Vallecito cuenta con personal en su mayoría entre 50 - 60 años. Cabe resaltar que para establecer conclusiones deberíamos considerar la diferencia en factores contribuyentes para el desarrollo del síndrome como el entorno, la carga familiar, entre otros.

La comparación con los resultados obtenidos en estudios similares no se establece del todo exacta y concluyente ya que, fueron realizados en grupos profesionales específicos a diferencia de la presente investigación que evaluó diferentes grupos profesionales en conjunto. Si bien esta variable cuenta con un instrumento validado nos brinda resultados que indicarían la presencia del síndrome; sin embargo, es importante el contraste clínico que podrían evidenciar "falsos positivos" (Castillo et al., 2015; Curiel et al., 2014; Hernández, 2015; Quiroz \& Saco, 2014), por lo que debemos tener en cuenta que los trabajadores con niveles altos en alguna subescala de este síndrome deben ser evaluados con mayor profundidad clínica.

La variable desempeño laboral mostró resultados altos y homogéneos en todo el personal de salud. Chumpitaz J. (2017) encontró valores bajos estableciendo una ineficiencia en su labor, estos resultados se asocian a la forma de evaluación y al uso de instrumentos especialmente diseñados que evaluaban el correcto desarrollo de procedimientos y técnicas inherentes al profesional. En la presente investigación se usó un cuestionario de autoevaluación lo que podría predisponer a sesgos, ya que el profesional evaluado puede direccionar sus respuestas.

Salas, Días, \& Perez (2015) muestran resultados donde el personal evaluado presenta dificultad para trabajar con los demás, susceptibilidad para corrección de errores, desacuerdo con el trabajo que realizan, falta de comunicación que repercute en la calidad del servicio, valores diferentes a los de esta investigación, esto se debe a que el C.S. Vallecito es un establecimiento pequeño y la interrelación entre trabajadores es constante, a diferencia del estudio citado que se llevó a cabo en un hospital donde las relaciones entre el personal no fluyen de la misma manera (Cortez \& García, 2015).

En relación a las escalas del desempeño laboral evaluadas la comparación con otras investigaciones no procede, por no contar con antecedentes donde se aplicó el mismo instrumento y sus escalas correspondientes; en estudios que evaluaron la misma variable consideraron el riesgo a sufrir despersonalización por las condiciones de trabajo y la carga laboral, así como la dificultad para el trabajo en equipo, entre otros ítems (Lavy \& Magdalena, 2017). Además en relación a los resultados del personal no profesional no se encontraron estudios similares ya que la evaluación del desempeño laboral se ha realizado mayormente en el personal de salud profesional (Sopla, 2018). 
Al evaluar ambas variables, se establece que no existe relación estadística con un coeficiente de correlación bajo, que podría asociarse a que los grupos de estudio (profesionales y no profesionales), no son grupos homogéneos. En otras investigaciones similares la relación fue estadísticamente significativa por la homogeneidad de la muestra y el uso de instrumentos más especializados sobre todo en los relacionados al desempeño laboral (De la Cruz, 2018; Mejía et al., 2019).

\section{CONCLUSIONES}

El personal de salud presenta síndrome de Burnout de nivel medio (7.09), a la evaluación por subescalas: el agotamiento emocional es de nivel bajo indica que el personal no está exhausto emocionalmente con su trabajo, la despersonalización de nivel medio evidencia un grado moderado de actitudes de frialdad y distanciamiento hacia los usuarios, la realización personal de nivel bajo con sentimientos de autoeficiencia y realización personal con su trabajo.

El desempeño laboral del personal de salud es alto (35.71); el cumplimiento de su labor es bastante homogéneo, a la evaluación por escalas: la calidad y técnicas de atención es eficiente, la capacitación y educación muestra un personal poco capacitado y actualizado, la productividad evidencia que se ajusta a las necesidades de los servicios que brinda con resultados óptimos, las aptitudes muestran su compromiso con su institución brindando una atención de calidad y oportuna a los usuarios de los servicios de salud.

El síndrome de Burnout y el desempeño laboral del personal de salud no presentan relación al establecerse una correlación estadística no significativa $(r=0.063)$.

\section{REFERENCIAS BIBLIOGRÁFICAS}

Aranda, C. (2014). El síndrome de Burnout o de quemarse en el trabajo: un conflicto en la salud de los profesionales. Revista de Investigación en Psicología, 14(2), 45. https://doi.org/10.15381/rinvp.v14i2.2099

Arias, W., \& Muñoz, A. (2016). Síndrome de burnout en personal de enfermería de Arequipa Síndrome de burnout en personal de enfermería de Arequipa. Revista Cubana de Salud Pública, 42(4).

Atance, J. C. (1997). Aspectos epidemiológicos del síndrome de Burnout en personal sanitario. Revista Española de Salud Pública, 71(3).

Ayquipa, J. C. (2017). Relación entre estrés laboral y funcionalidad familiar en trabajadores de atención primaria de la Red de Salud Arequipa. Universidad Nacional de San Agustin.

Caballero, C., Hederich, C., \& Palacio, J. E. (2014). El Burnout académico: delimitación del síndrome y factores asociados con su aparición. Revista Latinoamericana de Psicologia, 42(1), 131.

Carillo, R., Gómez, K., \& De lo Monteros, I. (2017). Síndrome de Burnout en la práctica médica. Medicina Interna de México, 28(6). 
Castillo, I., Jairo, C., \& Alvis, L. (2015). Síndrome de Burnout en el personal médico de una institución prestadora de servicios de salud de Cartagena de Indias. Revista de La Universidad Industrial de Santander. Salud, 47(2), 187-192.

Castro, A., Rodríguez, M., Moreno, C., Vicente, C., Arroyos, M., \& Fernández, M. (2016). Prevalencia del síndrome de Burnout en fisioterapia. Fisioterapia, 28(1), 17-22. https://doi.org/10.1016/S0211-5638(06)74017-2

Cavero, K., Condor, M., \& Ramos, E. (2017). Síndrome de Burnout en el profesional de enfermeria del servicio de la unidad de cuidados intensivos neonatales en una clínica, Lima 2017.

Chumpitaz, J. (2017). Evaluación del desempeño laboral según características demográficas del personal de enfermería del servicio Gineco-Obstetricia del Hospital de Ventanilla 2017. Universidad César Vallejo.

Chung, K., \& Salas, M. (2018). Nivel de estrés laboral y su relación con el desempeño profesional en enfermeros del servicio de emergencia- Hospital Regional Docente e Trujillo 2018. Universidad Privada Antenor Orrego.

Consuelo, C. (2018). Síndrome de Burnout y desempeño laboral del personal asistencial del Hospital de la Policía Nacional del Perú - Lima. Universidad Cesar Vallejo.

Contreras, S., Priego, H., Palacios, S., Ávalos, M., Morales, M., \& Córdova Hernández, J. A. (2015). Síndrome de Burnout, funcionalidad familiar y factores relacionados en personal médico y de enfermería de un hospital de Villahermosa, Tabasco, México. Horizonte Sanitario, 12(2), 45. https://doi.org/10.19136/hs.v12i2.238

Cortez, R., \& García, A. (2015). Mejoramiento del desempeño laboral de los trabajadores del sector salud: el caso de Nicaragua. Apuntes: Revista de Ciencias Sociales, 38(69), 81-100. https://doi.org/10.21678/apuntes.69.632

Curiel, J., Rodriguez, M., \& Guerrero, F. (2014). Síndrome de agotamiento profesional en personal de atención de salud. Rev Med Inst Mex Seguro Soc, 44(3), 221-226.

De la Cruz, Y. (2018). Estrés laboral y desempeño profesional servicio de emergencia Hospital nuestra Señora de las Mercedes Carhuaz 2016.

Del Río, O., Perezagua, M., \& Vidal, B. (2015). El síndrome de burnout en los enfermeros/as del Hospital de la Salud de Toledo. Enfermería en Cardiología, 28, 24-29.

Díaz, G., \& Machuca, S. (2018). Dimensiones del síndrome de Burnout y su presencia en el desempeño laboral del personal médico de la Red de salud Cajamarca 2017.

Do Carmo, M., Chaves, M., Marchi, R., Almeida, L., Oliveira, I., \& Pedrao, L. (2016). Exceso de trabajo y agravios mentales a los trabajadores de la salud. Revista Cubana de Enfermeria, 26(1). 
Gomero, R., Palomino, J., Ruíz, F., \& Yesán, L. (2015). El Síndrome de Burnout en personal sanitario de los hospitales de la empresa minera de Southern Perú Copper Corporation: estudio Piloto. Revista Médica Herediana, 16(4).

Gómez, I. (2017). Factores asociados al síndrome de Burnout en el personal de enfermeria del servicio de emergecia del Hospital San Juan de Lurigancho. Universidad María Auxiliadora - UMA.

Hederich-Martínez, C., \& Caballero-Domínguez, C. (2016). Validación del cuestionario Maslach Burnout Inventory-Student Survey (MBI-SS) en contexto académico colombiano Validation of Maslach Burnout Inventory-Student Survey (MBI-SS) in Colombian academic context. 9(1), 1-15. https://doi.org/10.21615/3511

Hernández, J. (2015). Estrés y Burnout en profesionales de la salud de los niveles primario y secundario de atención. Revista Cubana de Salud Pública, 29(2), 103-110.

Lavy, R., \& Magdalena, Z. (2017). Relación entre el nivel de estrés y el desempeño laboral del personal de enfermeria del Hospital apoyo Camaná 2015. Universidad Alas peruanas.

Leal, C., Díaz, J., -tirado, S., Rodriguez, J., \& C, V. (2015). Communication skills: a preventive factor in Burnout syndrome in health professionals. Anales Del Sistema Sanitario de Navarra, 38(2), 213-223. https://doi.org/10.23938/assn.0070

Mamani, A., Obando, R., Uribe, A., \& Vivanco, M. (2017). Factores que desencadenan el estrés y sus consecuencias en el desempeño laboral en emergencia. Rev Per Obst Enf., 3(1), 50-57.

Mansilla, F., \& Favieres Alejandra. (2016, June). El estrés laboral y su prevención.

Martinez, A. (2013). Sindrome De Burnout y desempeño laboral del enfermo en el Hospital Nacional Victor Larco Herrera Diciembre-2012. Universidad Ricardo Palma.

Mejía, P., Sany, M., \& Aguilar, N. (2019). Sobrecarga laboral y calidad de cuidado del profesional de enfermería desde la perspectiva del usuario.

Mendoza, H. (2014). Prevalencia del Síndrome de burnout en personal de enfermería de dos instituciones de salud. Rev Enferm Inst Mex Seguro Soc., 24(2), 115-122.

Mendoza, H. (2016). Factores asociados al síndrome de Burnout como herramienta de gestión en odontología. Universidad San Martín de Porres.

Muñoz, A., Aras, W., \& Caycho, T. (2019). Síndrome de burnout en médicos de la ciudad de Arequipa (Perú). Revista Chilena de Neuro-Psiquiatría, 57(2).

Ochante, F. de M., Pimentel, S., \& Tello, M. (2018). Estrés laboral de la enfermera y la calidad de atención percibido por el familiar en el servicio de pediatría en un hospital nacional. Lima 2017 - 2018. Universidad Cayetano Heredia.

Ortega, C., \& López, F. (2016). El Burnout o síndrome de estar quemado en los profesionales 
sanitarios: revisión y perspectivas. Internatonal Journal of Clinical and Health Psychology, 4(1).

Pérez, E. (2006). Síndrome de burnout como factor de riesgo de depresión en médicos residentes. Medicina Interna de Mexico, 22(4), 282-286.

Quirate, M., Carvajal, \& Almanza, J. (2017). Síndrome de Burnout en el personal de residentes de Ginecología y Obstetricia en el Hospital Militar de Especialidades de la Mujer y Neonatología. 67(6).

Quiroz, R., \& Saco, S. (2014). Factores Asociados al Sindrome Burnout en Médicos y Enfermeras del Hospital Nacional Sur Este de EsSalud del Cusco. Revista Semestral de la Facultad de Medicina Humana, 23(12), 11-22.

Rojas, E., Obregoón, G., Gonzales, M., Alvarado, S., Meneses, A., Riveros, A., \& Sanchez, J. (2016). Factores asociados con el síndrome de Burnout en el equipo médico y paramédico en el Instituto Nacional de Cancerología de México | Gaceta Mexicana de Oncología. Gaceta Mexicana de Oncología, 11(2), 72-81.

Rubio, J. (2016). Fuentes de estrés, Sindrome de Burnout y actitudes difuncionales de orientadores de un Instituto de Enseñanza Secundaria. Universidad de extremadura.

Salas, R., Días, L., \& Perez, H. (2015). Las competencias y el desempeño laboral en el Sistema Nacional de Salud. Revista Cubana de Educacion Medica Superior, 26(4), 604-617.

Sánchez, P., \& Sierra, V. (2014). Síndrome de Burnout en el personal de enfermería en UVI. Enfermeria Global, 13(1), 252-266. https://doi.org/10.6018/eglobal.13.1.176811

Solís, R., Tantalean, M., Burgos, R., \& Chamb, J. (2017). Agotamiento profesional: prevalencia y factores asociados en médicos y enfermeras en siete regiones del Perú. Anales de La Facultad de Medicina, 78(3), 270. https://doi.org/10.15381/anales.v78i3.13757

Sopla, V. (2018). Desempeño Laboral del Profesional de Enfermería del Hospital de Apoyo "Gustavo Lanatta Lujan”, Bahua y del Hospital de apoyo "Santiago Apostol”, Utcubamba, Amazona - 2017. 1-69.

Sostena, P. (2014). Desgaste profesional en los médicos de Atención Primaria de Barcelona. Medifam, 12(10), 17-25.

Terrones, J., Cisneros, V., \& Arreolaa, J. (2016). Síndrome de Burnout en residentes médicos del Hospital General de Durango, Mexico. Revista Médica Del Instituto Mexicano Del Seguro Social, 54(618), 242-248.

Thomaé, M., Ayala, E., Sphan, M., \& Stortti, M. (2015). Etiología y Prevención del Síndrome de Burnout en los trabajadores de la salud. Revista de Posgrado de la vía Cátedra de Medicina, $153,18-21$.

Valdivia, C. (2014). Clima organizacional en el desempeño laboral del personal. In Lexus (Vol. 4, 
Issue None). Universidad Nacional de Trujillo.

Vásquez, J., Maruy, A., \& Verne, E. (2015). Frecuencia del síndrome de Burnout y niveles de sus dimensiones en el personal de salud del servicio de emergencia de pediatría del Hospital Nacional Cayetano Heredia en el año 2014. Lima, Perú. Revista de Neuro-Psiquiatria, 77(3), 168. https://doi.org/10.20453/rnp.v77i3.2031

Vivanco, M., Sánchez, C., Maldonado, R., \& Erique, E. (2018). Síndrome de Burnout en profesionales de la salud en instituciones públicas y privadas: Un análisis en la provincia de Loja , Ecuador. Burnout syndrome in health professionals in public and private institutions : an analysis in the province of Loja, Ecuad. Revista Boletín REDIPE 7, 179-194.

Yaya, V., Jurado, L., \& Serrano, E. (2018). Estrés y desempeño laboral de los profesionales de enfermería del servicio de emergencia adulto de una clínica privada de Lima, 2017 . Universidad peruana Unión.

Yslado, R., Cermeño, B., Rodriguez, D., \& Sánchez, J. (2014). Síndrome de Burnout y factores sociodemográficos-organizacionales en profesionales de salud. Hospitales del callejón de Concuchos, Ancash, Perú. Revista IIPSI, 16, 199-209.

\section{Conflicto de intereses / Competing interests:}

Los autores declaran que no incurren en conflictos de intereses.

\section{Rol de los autores / Authors Roles:}

Claudia Lauracio: conceptualización, curación de datos, análisis formal, adquisición de fondos, investigación, metodología, administración del proyecto, recursos, software, supervisión, validación, visualización, escritura preparación del borrador original, escritura - revisar \& amp; edición.

Teófilo Lauracio: conceptualización, investigación, metodología, administración del proyecto, recursos, software, supervisión, validación, visualización, escritura - preparación del borrador original, escritura - revisar \& amp; edición.

\section{Fuentes de financiamiento / Funding:}

Los autores declaran que no recibieron un fondo específico para esta investigación.

\section{Aspectos éticos / legales; Ethics / legals:}

Los autores declaran no haber incurrido en aspectos antiéticos, ni haber omitido aspectos legales en la realización de la investigación. 